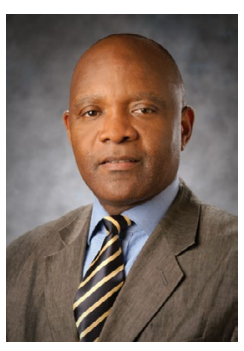

\title{
COVID-19: unprecedented but expected
}

\author{
The COVID-19 pandemic provides an opportunity to reimagine preparedness for and responses to \\ future pandemics.
}

T he COVID-19 pandemic has emphasized global connectivity, vulnerability and inequities.

The global response to the COVID-19 pandemic also revealed several fault lines: protectionism versus global cooperation, and politics versus public health. We must learn lessons about how the world responded to the COVID-19 pandemic, as it will not be the final pandemic to challenge the world.

About 103 years ago, in 1918, the world witnessed how catastrophic a global pandemic can be. The pandemic, caused by the Spanish flu, swept across the world as a devastating global public-health tragedy, as an estimated 50 million people died in 2 years. At that time, there were basically no technological countermeasures: no diagnostics, mechanical ventilators or antiviral agents.

Since that pandemic, important advances have been made in the areas of vaccines, treatments, preparedness and response. Although much progress has been made, preparing for the next pandemic will require that we review how our current health-security-governance architecture is structured and has evolved over the years.

As COVID-19 spread across the world, governments in different countries enacted a number of measures to safeguard their economies and health systems from the impact of the pandemic. However, as the pandemic deepened, and the economic downturn worsened, several countries used national-security and public-health exceptions to justify protectionism, which included restrictions on personal protective equipment and diagnostics and 'vaccine nationalism?.

Handling the COVID-19 pandemic demands an appreciation of the health consequences and also the social, political, security and economic consequences. This entails a balancing act between various interests and goals. While some countries took measures that were legitimate, other measures were simply due to political expedience. This divide between politics and public health also complicated global cooperation to effectively fight the pandemic and rescue the world from the global economic impact.
The new health-security architecture will require that the World Health Organization (WHO) be strengthened and empowered with the authority to quickly investigate the origin of disease outbreaks and manage access to new diagnostics, vaccines and therapeutics. In 1958, 10 years after the WHO was formally established, it undertook a global initiative to eradicate smallpox. At that time, smallpox was killing an estimated 2 million people per year. In 1979, smallpox was declared eradicated - the first disease in history to be eliminated by human effort. This historical success clearly shows how global cooperation and governance can have huge impact in safeguarding our health security. An empowered WHO should be enabled to effectively implement the international health regulations.

However, because the world's population has expanded considerably since the creation of the WHO, regional architectures such as the European Centre for Disease Prevention and Control and the Africa Centres for Disease Control and Prevention must be strengthened and given the authority to declare disease outbreaks as regional threats and coordinate responses. Global health security begins at the national level; therefore, we should build and strengthen science-led national public-health institutions across the world, with strong support for organizations such as the International Association of National Public Health Institutes.

In addition to reviewing our global health-security-governance architecture, we must enhance and invest in our technical capacities and capabilities. First, partnership with the private sector to invest in digital technology will be critical for fighting new pandemics and disease outbreaks. For instance, artificial intelligence, with its analytical power, will be a major asset in detecting patterns in the spread of pathogens or potential treatments. In fact, artificial intelligence is changing the way disease outbreaks are tracked and managed, aiding medical practitioners and saving lives. Moreover, other emerging digital technologies such as blockchain can also bring novel solutions and approaches to the way we fight future pandemics.
Second, strengthening global and regional infrastructure and novel platforms for the development and distribution of vaccines, diagnostics and therapy is vital. For instance, the present shortage of and limited access to vaccines against COVID-19 speaks to the need to have regional centers of excellence, such as the Serum Institute of India, that can manufacture vaccines rapidly for use in a pandemic. To achieve this goal, we need to build trust among nations to enhance the ability to rapidly share viral specimens and genomic sequences that are critical ingredients for research and development into vaccines, diagnostics and therapeutics.

Third, strengthening systems for the surveillance of viruses in birds and pigs should be a priority for preparing for and responding to the next pandemic, as $>70 \%$ of emerging diseases are linked to animal-to-human transmission. Continuous loss of natural habitats due to deforestation and the rapid growth of livestock farming is stressing animal populations and putting them into more-frequent contact with people.

Fourth, investment is needed in the development of the public-health workforce, particularly for field epidemiologists, frontline responders and community healthcare workers, especially in developing countries. In addition, there is an urgent need to develop capacity for behavioral scientists in pandemic responses.

In sum, the present COVID-19 pandemic is unprecedented but was expected. We must come out of this pandemic determined to do better in terms of preparedness than when we went into the pandemic; otherwise, we will have not learned any lessons.

\section{John N. Nkengasong ${ }^{\circledR}$}

Africa Centres for Disease Control and Prevention, Addis Ababa, Ethiopia.

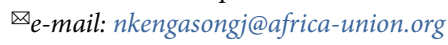

Published online: 15 March 2021

https://doi.org/10.1038/s41591-021-01269-x

Competing interests

The author declares no competing interests. 